\title{
Innate Immune System for Diagnostics and Therapy: Progress in Fundamental Knowledge and Clinical Application
}

\author{
Julia Kzhyshkowska Peter Bugert \\ Institute of Transfusion Medicine and Immunology, Medical Faculty Mannheim, Heidelberg University; German Red Cross Blood Service \\ Baden-Württemberg - Hessen; Mannheim, Germany
}

The innate immune system was evolutionary designed to protect the organism against various types of pathogens, including bacteria, parasites and viruses. However, in higher organisms the function of innate immune system is not restricted to the host defence and protection against of non-self components. The essential feature of the innate immune system that is required continuously for the haemostatic balance and healthy tissue turnover is the ability of major population of innate immune cells - tissue macrophages - to recognise and eliminate unwanted self components, including apoptotic cells, transformed cells, modified lipoproteins, excess of growth factors, cytokines and extracellular matrix components [1]. In case of pathogen attack or trauma, the innate immune system initiates acute inflammatory reaction and gives signals for adaptive immune cells to amplify the reaction in the antigen-specific ways. Once the danger is eliminated, the innate immune cells coordinate the resolution/healing phase and restore homeostatic balance. During the healing phase, the innate immune system supports angiogenesis, formation of tissue-specific extracellular matrix as well as recruitment and differentiation of somatic cells. The mistakes made by innate immune cells result in a broad range of pathologies, including cancer, cardio-metabolic disorders, allergies and neurodegeneration, and can also lead to the intolerance of implanted materials [2,3].

This issue is dedicated to demonstrate the recent advances in translation of fundamental knowledge about activation and function of the innate immune system into the development of new diagnostic and therapeutic approaches for several diseases with highest socio-economic significance: cardio-vascular disorders, airway inflammation and cancer.

Already in the early stage of these pathologies, innate immune cells undergo pathological activation that can be detected on the level of surface and secreted biomarkers. Circulating monocytes are primary sensors for changed levels of inflammatory cytokine, modified lipoproteins and hypoglycaemia in the blood circulation. Also activated platelets can induce switch towards CD16+ intermediate or non-classical monocyte formation. This and other mechanisms such as the release of cytokines by activated platelets or the interaction of platelets with immune cells highlight the significant role of platelets in the context of innate immunity [4]. Accumulating evidence in the identification of monocyte subsets by using not only CD16+ but also cytokine receptors, scavenger receptors and Toll-like receptors (TLRs) as biomarkers suggested that this is a very promising approach for the sensitive detection of hidden/lowgrade inflammation that precedes cardio-metabolic disorders [5]. Moreover, the individual patient's predisposition for detrimental responses can be detected sensitively by ex vivo challenging of primary monocytes. These results can be used, e.g., for the prediction of potential inflammatory responses to biomaterials allowing the individualised selection of suitable materials and procedures in regenerative medicine. However, targeting of specific monocyte subsets needs much deeper understanding of their specific functions and their potential to differentiate into subsets of mature macrophages. Functions as well as potentials of these monocyte subsets thus become a field of intensive investigation today.

The detrimental role of eosinophils, another type of immune cells, in inflammatory disorders, in particular asthma, is clearly proven [6]. Series of novel drugs and monoclonal antibodies that specifically inhibit eosinophils are under development or in clinical trials. Moreover, recent advances in our understanding of eosinophil biology as well as the identification of their complex role not only in detrimental inflammatory reactions but also in control of homeostasis indicate that therapeutic targeting of these cells is a promising tool in the treatment of a broad range of eosinophiliaassociated diseases. Targeting of innate immune receptors expressed on a broad range of cells is another efficient approach for treatment of airway inflammation. Recently, TLR7 expressed on

\section{KARGER}

Fax +497614520714
(๑) 2016 S. Karger GmbH, Freiburg

$1660-3796 / 16 / 0432-0063 \$ 39.50 / 0$
Prof. Dr. Julia Kzhyshkowska

Institut für Transfusionsmedizin und Immunologie, Medizinische Fakultät Mannheim, Universität Heidelberg

DRK-Blutspendedienst Baden-Württemberg - Hessen

Friedrich-Ebert-Straße 107, 68167 Mannheim, Germany

julia.kzhyshkowska@medma.uni-heidelberg.de 
plasmocytoid dendritic cells, monocytes, eosinophils, natural killer (NK) cells, T cells, epithelial cells and neurons attracted attention [7]. Thus synthetic TLR7 agonists are able to reduce airway hyperactivity, eosinophilic inflammation and airway remodelling. TLR7 ligands were suggested for treatment of both allergic and virusinduced airway disorders and have already entered clinical trials for treatment of allergic rhinitis [7].

The innate immune system has a highly complex role in progression of cancer, where several cell types including tumour-associated macrophages, neutrophils, mast cells and NK cells regulate primary tumour growth and metastatic processes by interacting with cancer cells, the tumour microenvironment and the vascular and lymphatic system [8-11]. The crucial step in tumour progression is the angiogenic switch, where for a long time tumour-associated macrophages were believed to be key inducing cells [12]. In this issue, Ammendola et al. [13] highlighted recent advances in the undertraining of the mechanisms of stimulation of tumour angiogenesis by mast cells and discuss new anti-cancer therapeutic strategies based on targeting mast cell degranulation factors.

Another essential process that has to be considered in developing novel anti-cancer therapies is tumour necrosis that occurs in hypoxic conditions and is associated with the release of damageassociated molecular pattern molecules (DAMPs). DAMPs act as 'danger signals', recruiting inflammatory cells and promoting chronic inflammation and cancer progression. Lotfi et al. [14] suggest that the oxidizing conditions should be considered for therapeutic targeting of the tumour microenvironment, where adoptive transfer of eosinophils or induction of eosinophilia in tumour patients may be helpful. Suck et al. [15] elucidated another emerging cell therapy approach which is applied in the treatment of leukaemia and is based on the application of stable engineered NK cell lines. This approach is promising for the standardisation of leukaemia treatment and potentially can also be used for treatment of solid tumours. However, the risks associated with clinical application of artificially activated NK cells still require very careful evaluation.

In summary, targeting of innate immune cells, individual innate immune molecules and applying specifically programmed innate immune cells open new horizons in the treatment of a spectrum of disorders where chronic inflammation is a driving force. However, in order to increase efficiency and reduce the inherited risks of such approaches, a fundamental knowledge about the interplay of different innate immune cells and their cross-talk with adaptive immune cells, with the local microenvironment, with the vascular network and somatic cells in humans is indispensable.

\section{References}

1 Gordon S, Plüddemann A, Martinez Estrada F: Macrophage heterogeneity in tissues: phenotypic diversity and functions. Immunol Rev 2014;262:36-55.

2 Kzhyshkowska J, Neyen C, Gordon S: Role of macrophage scavenger receptors in atherosclerosis. Immunobiology 2012;217:492-502.

3 Wynn TA, Chawla A, Pollard JW: Macrophage biology in development, homeostasis and disease. Nature 2013; 496:445-455.

4 Kral JB, Schrottmaier WC, Salzmann M, Assinger A: Platelet interaction with innate immune cells. Transfus Med Hemother 2016;43: DOI: 10.1159/000444807.

5 Kzhyshkowska J, Gudima A, Moganti K, Gratchev A, Orekhov A: Perspectives for monocyte/macrophagesbased diagnostics of chronic inflammation. Transfus Med Hemother 2016;43: DOI: 10.1159/000444943.
6 Long H, Liao W, Wang L, Lu Q: A player and coordinator: the versatile roles of eosinophils in the immune system. Transfus Med Hemother 2016;43: DOI: $10.1159 / 000445215$.

7 Lebold KM, Jacoby DB, Drake MG: Toll-like receptor 7-targeted therapy in respiratory disease. Transfus Med Hemother 2016;43: DOI: 10.1159/000445324.

8 Riabov V, Gudima A, Wang N, Mickley A, Orekhov A, Kzhyshkowska J: Role of tumor associated macrophages in tumor angiogenesis and lymphangiogenesis. Front Physiol 2014;5:75.

9 Liang W, Ferrara N: The complex role of neutrophils in tumor angiogenesis and metastasis. Cancer Immunol Res 2016;4:83-91.

10 Ribatti D: Mast cells as therapeutic target in cancer. Eur J Pharmacol 2015;pii:S0014-2999(15)00356-8.
11 Iannello A, Thompson TW, Ardolino M, Marcus A, Raulet DH: Immunosurveillance and immunotherapy of tumors by innate immune cells. Curr Opin Immunol 2016;38:52-58.

12 Noy R, Pollard JW: Tumor-associated macrophages: from mechanisms to therapy. Immunity 2014;41:49-61.

13 Ammendola M, Sacco R, Sammarco G, Luposella M, Patruno R, Gadaleta CD, De Sarro G, Ranieri G: Mast cell targeted strategies in cancer therapy. Transfus Med Hemother 2016;43: DOI: 10.1159/000444942.

14 Lotfi R, Kaltenmeier C, Lotze MT, Bergmann C: Until death do us part: necrosis and oxidation promote the tumor microenvironment. Transfus Med Hemother 2016;43: DOI: 10.1159/000444941.

15 Suck G, Linn YC, Tonn T: Natural killer cells for therapy of leukemia. Transfus Med Hemother 2016;43: DOI: $10.1159 / 000445325$. 